\title{
Global Observability of the Steady State Generator in Output Regulation Problem
}

\author{
Zhiyong Chen
}

\begin{abstract}
An output regulation problem can be converted into a stabilization problem thanks to an appropriately designed internal model. In literature, the successful construction of an internal model relies on the linear observability condition of the steady state generator. This paper defines the global observability property of the steady state generator, under which the output regulation problem is solved for a more general class of systems.
\end{abstract}

\section{INTRODUCTION}

The robust output regulation problem is concerned with designing a control law for an uncertain plant such that the closed-loop system satisfies two requirements. The first one is the closed-loop stability, and the second one is the asymptotic tracking and disturbance rejection, i.e., to have the output of the closed-loop system asymptotically track a class of reference inputs in the presence of a class of disturbances. Here both the reference inputs and disturbances are generated by an autonomous exosystem. This problem for linear systems can be traced back to 1970s in [2], [3], and [4], among others. A salient outcome of this research is the internal model principle which enables the conversion of the output regulation problem into an eigenvalue placement problem for an augmented linear system. In the last two decades, the robust output regulation problem for nonlinear systems was pursued by quite a few people in [1], [7], [8], [11], and [13], from locally, semi-globally to globally. Various solvability conditions have been given which impose assumptions on the solution of the regulator equations. An analogue development for nonlinear systems is that an internal model enables the conversion of the robust regulation problem into a robust stabilization problem for an augmented system. In particular, a systematic framework on this conversion was developed in [7].

The crucial step of applying the systematic framework is the design of an appropriate internal model. Essentially, an internal model works as an observer of the steady state generator which reproduces the steady state/input. Therefore, the successful design of an internal model relies on a certain observability condition of the steady state generator. The condition was first studied in [8] which requires the solution of the regulator equations be a polynomial in the exogenous signal. Later, a different immersion condition was given in

Z. Chen is with School of Electrical Engineering and Computer Science, The University of Newcastle, Callaghan, NSW 2308, Australia, zhiyong. chen@newcastle.edu.au.

The work was partially supported by the Australian Research Council under grant No. DP0878724.
[1]. It was further shown in [5] that, either the polynomial or the immersion condition implies a trigonometric polynomial condition. Essentially, all three conditions are equivalent, and they guarantee the existence of a linear observable steady state generator.

A milder assumption that allows the solution of the regulator equations to be non-polynomial nonlinearity was given in [7]. With this assumption, a nonlinear steady state generator is given for reproducing the steady state/input. Motivated by the linear case, an internal model is designed under the linear observability assumption on the steady state generator. It is also because only the linear observability is examined that a certain nonlinear term remains in the internal model. This nonlinear residue may cause some controller design difficulties which will be discussed in this paper. It has been known that the fundamental mechanism for the robust output regulation is the conversion into a robust stabilization problem for an augmented system. Specifically, the aforementioned nonlinear residue may complicate the stabilizer design for the resulted augmented system. In the existing results, the nonlinear term is assumed to be globally Lipschitz with a given Lipschitz constant (see, e.g. [7]). In other words, the nonlinear term in the internal model is assumed to be dominated by the linear vector field. Therefore, the stability property of the internal model can be dominated by its linearization. It turns out that this stability property is necessary for the solvability of the stabilization problem for the augmented system. Otherwise, when the incremental rate of the nonlinear residue doesn't satisfy the Lipschitz condition, the stabilization problem of the augmented system, or the output regulation problem of the original system, is not solved.

This paper aims to find a condition under which we can get rid of the nonlinear residue in the internal model. We have revealed that the nonlinear residue exists because the internal model is designed on the linearly observable part of the steady state generator. This observation motivates us to look for the global observability property of the steady state generator. In this paper, we define a global observability property, and further construct a linear internal model based on the globally observable steady state generator. As a result, the incremental assumption on the nonlinear residue become trivial. In this sense, the robust output regulation can be studied for a more general systems with non-polynomial nonlinearities.

The remaining sections are organized as follows. In Section II, we give the problem formulation and revisit the linear and nonlinear steady state generators with linear observ- 
ability. In Section III, the global observability/detectability condition is defined under which the global robust output regulation problem for a class of nonlinear systems is solved. A numerical example is provided in Section IV, followed by some concluding remarks in Section V.

\section{Problem Description and Preliminaries}

Consider a reference tracking and disturbance rejection problem of a nonlinear system with an affine input described as follows:

$$
\begin{aligned}
\dot{\xi} & =\phi(\xi, v, w)+b(w) u \\
e & =\xi-q(v, w) .
\end{aligned}
$$

In the system (2.1), $\xi \in \mathbb{R}^{n}$ is the state, $u \in \mathbb{R}^{n}$ the input, $e \in \mathbb{R}^{n}$ the output representing the tracking error, $v \in \mathbb{R}^{q}$ the exogenous signal representing the disturbance and/or the reference input, and $w \in \mathbb{R}^{s}$ the unknown parameter vector. The sign of $b(w) \in \mathbb{R}$ is known. In particular, we assume $\bar{b}>b(w)>\underline{b}$ for a positive numbers $\bar{b}$ and $\underline{b}$. The exogenous signal $v$ is produced by an autonomous system described by

$$
\dot{v}=A v
$$

where the matrix $A$ is neutrally stable, that is, all the eigenvalues of $A$ are simple with zero real parts. The control objective is to design an error output controller such that the states of the closed-loop system are bounded, and the tracking error asymptotically approaches to zero. This control problem is concerned with asymptotic tracking and disturbance rejection in addition to stability. However, for the class of system (2.1) we can incorporate the tracking objective into the disturbance rejection by using a simple coordination transformation $x=e$. In the new coordinate, the system (2.1) can be rewritten as follows:

$$
\begin{aligned}
\dot{x} & =f(x, v, w)+b(w) u \\
e & =x .
\end{aligned}
$$

We assume all functions in the setup are sufficiently smooth. Now, the problem studied in this paper can be formulated as follows.

Global Robust Output Regulation Problem (GRORP): Consider the system (2.3), for given compact sets $\mathbb{V} \subset \mathbb{R}^{q}$ and $\mathbb{W} \subset \mathbb{R}^{s}$, find a state $(x)$ feedback controller such that the closed-loop system satisfies the following two properties:

(i) The trajectories of the closed-loop system starting from any initial states exist and are bounded for all $t>0$;

(ii) The trajectories described in (i) also satisfy $\lim _{t \rightarrow \infty} e(t)=0$.

Remark 2.1: The first solvability condition for the GRORP is the existence of steady state/input. The steady state is clearly defined as $\mathbf{x}(v, w)=0$ due to the aforementioned property (ii). The steady input is determined by the equation

$$
0=f(0, v, w)+b(w) \mathbf{u}(v, w) .
$$

The above equation (2.4), together with $\mathbf{x}(v, w)=0$, are called the regulator equations. The functions $\mathbf{x}(v, w)$ and $\mathbf{u}(v, w)$ define the steady state and steady input, respectively. Their solvability of the regulator equations is a necessary condition for the solvability of the output regulation problem [9]. From (2.4), if $b(w)>\underline{b}$, we have, $\mathbf{u}(v, w)=$ $-b^{-1}(w) f(0, v, w)$.

To render the solvability of GRORP, an additional condition must be imposed on the solution of (2.4), i.e., $\mathbf{u}(v, w)$. Before discussing the conditions, we cite the definition of steady state generator as follows (see [7]).

Definition 2.1: The nonlinear system (2.2)-(2.3) is said to have a steady-state generator (SSG) with the output $y=$ $\mathbf{u}(v, w)$, if there exists a triple $\{\theta, \alpha, \beta\}$, where $\theta: \mathbb{R}^{q+s} \mapsto$ $\mathbb{R}^{r}, \alpha: \mathbb{R}^{r} \mapsto \mathbb{R}^{r}$, and $\beta: \mathbb{R}^{r} \mapsto \mathbb{R}^{n}$ for some integer $r$ are sufficiently smooth functions vanishing at the origin, such that, for all trajectories $v(t)$ of the exosystem, and $w \in \mathbb{R}^{s}$,

$$
\begin{aligned}
\dot{\theta}(v, w) & =\alpha(\theta(v, w)) \\
y & =\beta(\theta(v, w)) .
\end{aligned}
$$

\section{A. A linear SSG}

The first condition is given in [8] which requires the solution of the regulator equations be a polynomial in $v$ with its coefficients depending, in general, on $w$. Later, a different condition is given in [1] as follows. There exists a real vector $a \in \mathbb{R}^{r}$ such that (to save the notation, we assume $n=1$ in the following expression)

$$
\begin{array}{r}
L_{A v}^{r} \mathbf{u}(v, w)-a_{1} \mathbf{u}(v, w)-a_{2} L_{A v} \mathbf{u}(v, w)-\cdots \\
-a_{r} L_{A v}^{r-1} \mathbf{u}(v, w)=0
\end{array}
$$

where

$$
\begin{aligned}
L_{A v} \mathbf{u}(v, w) & =\frac{\partial \mathbf{u}(v, w)}{\partial v} A v \\
L_{A v}^{l} \mathbf{u}(v, w) & =\frac{\partial L_{A v}^{l-1} \mathbf{u}(v, w)}{\partial v} A v, l=2,3, \cdots .
\end{aligned}
$$

Also, it was shown that (2.6) holds if $\mathbf{u}(v, w)$ is a polynomial in $v$. It was further shown in [5] that, either the polynomial condition or (2.6) implies that $\mathbf{u}(v(t), w)$ is a trigonometric polynomial of $t$. Thus, essentially, all three conditions are equivalent, and they guarantee the existence of a linear steady state generator.

To give the linear steady state generator, we define some notations here. Let $\pi(v(t), w)$ be a trigonometric polynomial of $t$ or a polynomial in $v$, and $r$ the degree of its minimal zeroing polynomial, define a notation

$$
\mathcal{L} \pi(v, w)=\operatorname{col}\left(\pi(v, w), \dot{\pi}(v, w), \cdots, \frac{d^{r-1} \pi(v, w)}{d t^{r-1}}\right) .
$$

It should be noted that the operator $\mathcal{L}$ is defined along the specific exosystem (2.2). Now, let $\pi(v, w)=\mathbf{u}(v, w)$, and $\tau(v, w)=\mathcal{L} \pi(v, w)$, we have a linear steady state generator

$$
\dot{\tau}=\Phi \tau, \quad y=\Psi \tau
$$

where $\Phi$ is the companion matrix of the minimal zeroing polynomial of $\pi(v, w)$, and $\Psi=\left[\begin{array}{llll}1 & 0 & \cdots & 0\end{array}\right]$. It is clear that the pair $(\Psi, \Phi)$ is observable. 


\section{B. A linearly observable SSG}

The following condition is first introduced in [7] which is much milder than the aforementioned three conditions. It allows a nonlinear steady state generator.

Assumption 1: There exist polynomials $\pi_{1}(v, w)$, $\cdots, \pi_{\ell}(v, w)$ and a sufficiently smooth function $\rho$ vanishing at the origin such that, for all trajectories $v(t)$ of the exosystem, and $w \in \mathbb{R}^{s}$,

$$
\begin{aligned}
\mathbf{u}(v, w) & =\rho(\tau(v, w)) \\
\tau(v, w) & :=\operatorname{col}\left(\mathcal{L} \pi_{1}(v, w), \cdots, \mathcal{L} \pi_{\ell}(v, w)\right) .
\end{aligned}
$$

Under Assumption 1, the system (2.3) has a nonlinear steady state generator

$$
\dot{\tau}=\Phi \tau, \quad y=\rho(\tau)
$$

where

$$
\Phi=\text { block } \operatorname{diag}\left\{\Phi_{1}, \cdots, \Phi_{\ell}\right\}
$$

with $\Phi_{i}$ being the companion matrix of the minimal zeroing polynomial of $\pi_{i}(v, w)$. Let $\Psi$ be the Jacobian matrix of $\rho$ at the origin and

$$
\varrho(x):=\rho(x)-\Psi x
$$

be the nonlinear part of $\rho(x)$. In literature, a controller for the robust output regulation problem is designed under the linear observability and nonlinear incremental conditions as listed below.

(i) (Linear Observability Condition) The steady state generator (2.9) is linearly observable, i.e., the pair $(\Psi, \Phi)$ is observable.

Remark 2.2: (i) Below we describe the observability and detectability conditions in an alternative way. The descriptions will be further extended to the global observability and detectability conditions in the next section. The pair $(\Psi, \Phi)$ is observable if and only if for any Hurwitz matrix $M$, there exists a matrix $N$, such that, the Sylvester Equation:

$$
T \Phi-M T=N \Psi
$$

admits a nonsingular solution $T$. The pair $(\Psi, \Phi)$ is detectable if and only if there exists a pair $(M, N)$ with $M$ Hurwitz, such that the Sylvester Equation (2.10) admits a nonsingular solution $T$. Clearly, the observability condition is stronger than the detectability condition. But, in a controller design problem, it may suffice to have the latter.

(ii) We note that, for an arbitrary pair $(M, N)$ with $M$ Hurwitz, the Sylvester Equation (2.10) always has a solution $T$ since $\Phi$ and $M$ have disjoint spectrum. Moreover, for a single input system, if $(M, N)$ is controllable, and $(\Psi, \Phi)$ is observable, the solution $T$ to the Sylvester Equation is nonsingular.

(iii) Let $\Psi=\left[\Psi_{1}, \cdots, \Psi_{\ell}\right]$ with $\Psi_{i}$ having the dimension of $\mathcal{L} \pi_{i}(v, w)$. We have proved in [7] that if the polynomials $\pi_{i}(v, w)$ 's are pairwise coprime, and the pairs $\left(\Psi_{i}, \Phi_{i}\right)$ 's are observable, then $(\Psi, \Phi)$ is observable. (ii) (Nonlinear Incremental Condition) For the matrices $M, N, T$ used in equation (2.10), the function @ satisfies

$$
\begin{array}{r}
-2 z^{\top} P N\left[\varrho\left(T^{-1}(z+d)\right)-\varrho\left(T^{-1} d\right)\right] \\
\leq(1-\delta)\|z\|^{2}, \quad \forall z, d
\end{array}
$$

for some positive number $\delta$. The matrix $P$ is the solution to the Lyapunov Equation $P M+M^{\top} P=-I$.

Remark 2.3: The inequality (2.11) puts an incremental constrain on the nonlinear function $\varrho$. We note that this condition holds when

$$
\left|\varrho\left(T^{-1}(z+d)\right)-\varrho\left(T^{-1} d\right)\right| \leq(1-\delta)\|z\| /(2\|P N\|) .
$$

Thus, (2.11) holds if $\varrho$ is globally Lipschitz with a Lipschitz constant $L<\|T\| /(2\|P N\|)$.

With the linear observability condition, it is ready to revisit the framework of converting the GRORP into a global robust stabilization problem. With the notations

$$
\theta(v, w):=T \tau(v, w), \beta(\theta):=\rho\left(T^{-1} \theta\right),
$$

we can define an alternative steady state generator:

$$
\dot{\theta}(v, w)=T \Phi T^{-1} \theta(v, w), \quad y=\beta(\theta),
$$

and hence, an internal model candidate

$$
\dot{\eta}=M \eta+N u-N \psi(\eta) .
$$

In (2.12), we use

$$
\psi(\eta):=\beta(\eta)-\Psi T^{-1} \eta,
$$

or equivalently

$$
\psi(\eta):=\varrho\left(T^{-1} \eta\right),
$$

which is the nonlinear residue of $\beta(\eta)$. Conducting the coordinate transformation

$$
\begin{aligned}
\bar{u} & =u-\beta(\eta) \\
z & =\eta-\theta-N b^{-1}(w) x
\end{aligned}
$$

on (2.3) and (2.12) gives

$$
\begin{aligned}
\dot{x} & =f(x, v, w)-f(0, v, w)+b(w) \hbar(x, z, v, w)+b(w) \bar{u} \\
\dot{z} & =M z-N \psi(z+d)+N \psi(d)+\ell(x, v, w)
\end{aligned}
$$

with the notations defined as follows:

$$
\begin{aligned}
\hbar(x, z, v, w):= & \beta\left(z+N b^{-1}(w) x+\theta\right)-\beta(\theta) \\
\ell(x, v, w):= & -N \psi\left(N b^{-1}(w) x+\theta\right)+N \psi(\theta) \\
& +M N b^{-1}(w) x-N b^{-1}(w) f(x, v, w) \\
& +N b^{-1}(w) f(0, v, w) \\
d:= & N b^{-1}(w) x+\theta .
\end{aligned}
$$

In [7], we have proven that the GRORP is solved if the global robust stabilization problem of the augmented system (2.13) is solved. In the above development, because only a linear observability condition is examined on the steady state generator (2.9), we have to keep a nonlinear residue $\psi(\eta)$ in 
the internal model (2.12). In fact, the internal model, with $\eta=\theta, u=\beta(\theta)$, is required to satisfy

$$
T \Phi T^{-1} \theta=M \theta+N \beta(\theta)-N \psi(\theta) .
$$

The nonlinear residue $\psi$ is further passed to the $z$-subsystem in (2.13). It may cause some trouble in solving the stabilization of (2.13). Therefore, the nonlinear incremental condition has to be imposed on $\psi$ such that the stability property of the $z$-subsystem (in particular, the $z$-subsystem is robustly input-to-state stable with state $z$ and input $\ell$ ) is dominated by the linear $M z$ term (with a Hurwitz matrix $M$ ). As we will see in the example, if the inequality is violated, the $z$-subsystem doesn't have the stability property, which becomes an obstacle in solving the stabilization problem of (2.13).

\section{A Globally Observable SSG}

In last section, we revisit the internal model design procedure based on the linear observability/detectability condition of the steady state generator. But, we are aware of a difficulty caused by the nonlinear residue in the internal model. In this section, we aim to define a global observability/detectability condition, under which a linear internal model is successfully constructed. Under the Assumption 1, we have a nonlinear steady state generator (2.9), the global observability and detectability conditions of (2.9) are given below, which are motivated by the local counterparts in Remark 2.2.

Definition 3.1: The system (2.9) is said to be globally observable if for any Hurwitz matrix $M$, there exists a matrix $N$, such that, the differential equation

$$
\frac{\partial H(\tau)}{\partial \tau} \Phi \tau=M H(\tau)+N \rho(\tau)
$$

admits a diffeomorphic solution $H(\tau): \mathbb{R}^{r} \mapsto \mathbb{R}^{r}$.

The system (2.9) is said to be globally detectable if there exists a pair $(M, N)$ with $M$ Hurwitz, such that the differential equation (3.1) admits a diffeomorphic solution $H(\tau): \mathbb{R}^{r} \mapsto \mathbb{R}^{r}$.

Remark 3.1: If the system (2.9) is linearly observable/detectable, the equation (3.1) holds with the linear part of $\rho(\tau)$, i.e.,

$$
\frac{\partial H(\tau)}{\partial \tau} \Phi \tau=M H(\tau)+N \Psi \tau .
$$

Let $T$ be the Jacobian matrix of $H(\tau)$ at a point $\tau=0$, this equation further reduces to the Sylvester Equation (2.10). When $H(\tau)$ is a diffeomorphism, its Jacobian matrix $T$ is nonsingular.

Under Assumption 1, if the steady state generator (2.9) is global observable/detectable, we can design a linear internal model as follows.

First, with the notations

$$
\theta(v, w):=H(\tau(v, w)), \quad \beta(\theta)=\rho \circ H^{-1}(\theta),
$$

we can define another steady state generator

$$
\dot{\theta}=M \theta+N y, y=\beta(\theta) \text {. }
$$

And a linear internal model is constructed based on (3.2) as follows:

$$
\dot{\eta}=M \eta+N u .
$$

Conducting the coordinate transformation

$$
\begin{aligned}
& \bar{u}=u-\beta(\eta) \\
& \bar{\eta}=\eta-\theta
\end{aligned}
$$

on (2.3) and (3.3) gives

$$
\begin{aligned}
\dot{x}= & f(x, v, w)-f(0, v, w)+b(w) \beta(\eta)-b(w) \beta(\theta) \\
& +b(w) \bar{u} \\
\dot{\bar{\eta}}= & M \bar{\eta}+N(\bar{u}+\beta(\eta)-\beta(\theta))
\end{aligned}
$$

With another coordinate transformation

$$
z=\bar{\eta}-N b^{-1}(w) x
$$

the system (3.4) is rewritten as follows:

$$
\begin{aligned}
\dot{x} & =f(x, v, w)-f(0, v, w)+b(w) \hbar(x, z, v, w)+b(w) \bar{u} \\
\dot{z} & =M z+\ell(x, v, w)
\end{aligned}
$$

with

$$
\begin{aligned}
\hbar(x, z, v, w)= & \beta\left(z+N b^{-1}(w) x+\theta\right)-\beta(\theta) \\
\ell(x, v, w)= & M N b^{-1}(w) x-N b^{-1}(w) f(x, v, w) \\
& +N b^{-1}(w) f(0, v, w) .
\end{aligned}
$$

Remark 3.2: We note that under the global observability/detectablity condition, the nonlinear residue $\psi(\eta)$ disappears in the internal model (3.3) or the $z$-subsystem in (3.5). In other words, the internal model goes back to the so called canonical form used in [12] and [13]. It should be noted that the existing linear internal models were constructed under the linear steady state generator condition (existence of (2.7)) as we discussed in Section II.A. However, the linear internal model (3.3) is constructed based on the nonlinear steady state generator (2.9) allowing non-polynomial uncertainties in the system (2.3).

Next, we will give the solvability condition for the stabilization problem of the system (3.5), and hence for the GRORP of the original system (2.3). In this paper, we don't put emphasis on the stabilization part. If fact, there has been much progress on the global robust stabilization problem of a variety classes of nonlinear systems. Here we only point out that if the global robust stabilization of the original system (2.3) is solvable, then, the GRORP problem of (2.3) is also solvable. We note the former is a special case of the latter. Specifically, we assume the global stabilization problem of the original system (2.3) is solvable in the following sense by straightforwardly cancelling the steady input using a feedforward compensator.

Assumption 2: For the system (2.3), there exists a global stabilizer $u=\kappa(x)+\mathbf{u}(v, w)$ with a sufficiently smooth function $\kappa(\cdot)$ satisfying $\kappa(0)=0$. In particular, there exists a $C^{1}$ function $U_{x}(\cdot)$ satisfying $\underline{\gamma}(\|x\|) \leq U_{x}(x) \leq$ 
$\bar{\gamma}(\|x\|)$ for some class $\mathcal{K}_{\infty}$ functions $\gamma(\cdot)$ and $\bar{\gamma}(\cdot)$, such that, along the trajectory of the closed-loop system,

$$
\frac{d U_{x}(x)}{d t} \leq-\chi(x)
$$

for class $\mathcal{K}_{\infty}$ function $\chi(\cdot)$ satisfying

$$
\lim _{s \rightarrow 0^{+}} \sup \left(\chi^{-1}\left(s^{2}\right) / s\right)<+\infty \text {. }
$$

Under the Assumption 2, if the steady state term $\mathbf{u}(v, w)$ is exactly known, the GRORP is trivial by cancelling the steady input term using a feedforward compensator. The internal model technique is mainly used for handling the unknown term $\mathbf{u}(v, w)$. The main result is summarized in the following Theorem.

Theorem 3.1: Under Assumptions 1-2, if the steady state generator (2.9) is globally observable/detectable, there exists a sufficiently smooth function $\hat{u}(x)$ such that the GRORP for the system (2.3) is solved by the controller:

$$
u=\beta(\eta)+\kappa(x)+\hat{u}(x), \quad \dot{\eta}=M \eta+N u .
$$

Proof: Clearly, the nonlinear functions defined in (3.5) satisfy $\hbar(0,0, v, w)=0$ and $\ell(0, v, w)=0$ for all $v$ and $w$. As a result, we have for $v \in \mathbb{V}$ and $w \in \mathbb{W}$,

$$
\begin{aligned}
\|\hbar(x, z, v, w)\| & \leq\|x\| \hbar_{x}(x)+\|z\| \hbar_{z}(z) \\
\|\ell(x, v, w)\| & \leq\|x\| \ell_{x}(x)
\end{aligned}
$$

for some smooth nonnegative functions $\hbar_{x}, \hbar_{z}(x)$ and $\ell_{x}$.

Let $U_{z}(z)=2 z^{\top} P z$, and its derivative along the system (3.5) is

$$
\begin{aligned}
\frac{d U_{z}(z)}{d t} & \leq 2\left[-\|z\|^{2}+2 z^{\top} P \ell(x, v, w)\right] \\
& \leq-\|z\|^{2}+\|x\|^{2} \omega^{2}(x)
\end{aligned}
$$

for $\omega(x):=2\|P\| \ell_{x}(x)$. Hence, using the changing supply function technique [14], there exists a $V_{z}(x)$ such that,

$$
\frac{d V_{z}(z)}{d t} \leq-\|z\|^{2}\left(1+\hbar_{z}^{2}(z)\right)+\|x\|^{2} \varpi^{2}(x) .
$$

Next, let $\Delta(x):=1+\varpi^{2}(x)+\hbar_{x}^{2}(x)$. From (3.6), there exists $S(x)$ such that (see [10])

$$
S(x) \chi(x) \geq \Delta(x)\|x\|^{2} .
$$

Using the changing supply function technique again, there exists a $V_{x}(x)$, such that, along

$$
\dot{x}=f(x, v, w)-f(0, v, w)+b(w) \kappa(x),
$$

its derivative satisfies

$$
\frac{d V_{x}(x)}{d t} \leq-S(x) \chi(x) \leq-\Delta(x)\|x\|^{2} .
$$

Let $\bar{u}=\kappa(x)+\hat{u}(x)$ with $\hat{u}(x)$ to be determined later. Then, along

$$
\begin{array}{r}
\dot{x}=f(x, v, w)-f(0, v, w)+b(w) \hbar(x, z, v, w) \\
+b(w) \kappa(x)+b(w) \hat{u},
\end{array}
$$

we have

$$
\begin{aligned}
\frac{d V_{x}(x)}{d t} \leq & -\Delta(x)\|x\|^{2}+1 / 2\left\|\frac{\partial V_{x}(x)}{\partial x} b(w)\right\|^{2} \\
& +\|x\|^{2} \hbar_{x}^{2}(x)+\|z\|^{2} \hbar_{z}^{2}(z)+\frac{\partial V_{x}(x)}{\partial x} b(w) \hat{u}
\end{aligned}
$$

Finally, it suffices to choose

$$
\hat{u}(x)=-1 / 2 \bar{b}\left[\frac{\partial V_{x}(x)}{\partial x}\right]^{\top}
$$

such that

$$
1 / 2\left\|\frac{\partial V_{x}(x)}{\partial x} b(w)\right\|^{2}+\frac{\partial V_{x}(x)}{\partial x} b(w) \hat{u} \leq 0 .
$$

As a result, we have

$$
\frac{d\left(V_{z}(z)+V_{x}(x)\right)}{d t} \leq-\|z\|^{2}-\|x\|^{2}
$$

and the closed-loop system is globally asymptotically stable (GAS).

\section{AN EXAMPLE}

In this section, we give a numerical example to illustrate the effectiveness of the controller proposed in Theorem 3.1. For a 2-dimensional nonlinear system

$$
\begin{aligned}
\dot{\xi} & =\left[\begin{array}{c}
v_{2} \cos \xi_{2}-6 v_{1} \\
\sin \xi_{2}-\xi_{1}
\end{array}\right]+u \\
e & =\xi-\left[\begin{array}{ll}
v_{2} & v_{1}
\end{array}\right]^{\top}
\end{aligned}
$$

our objective is to design an error feedback controller to make the tracking error $e$ asymptotically approach to zero. The sinusoidal exogenous signal $v$ is produced by an exosystem (2.2) with

$$
A=\left[\begin{array}{cc}
0 & 1 \\
-4 & 0
\end{array}\right] \text {. }
$$

Letting $x=e$, the error system can be written as follows:

$$
\dot{x}=\left[\begin{array}{c}
v_{2} \cos \left(x_{2}+v_{1}\right)-2 v_{1} \\
\sin \left(x_{2}+v_{1}\right)-x_{1}-2 v_{2}
\end{array}\right]+u .
$$

First, we have the steady input $\mathbf{u}(v)$ from (4.1) satisfying Assumption 1. In particular, we have $\mathbf{u}(v)=\rho(\tau(v))$ with

$$
\rho(\tau)=\left[\begin{array}{c}
2 \tau_{1}-\tau_{2} \cos \tau_{1} \\
2 \tau_{2}-\sin \tau_{1}
\end{array}\right], \quad \tau(v)=v .
$$

Now, we have a steady state generator (2.9) with $\Phi=A$ and

$$
\Psi=\left[\begin{array}{cc}
2 & -1 \\
-1 & 2
\end{array}\right]
$$

It is clear that (2.9) is linearly observable. Specifically, pick a pair of matrices

$$
M=\left[\begin{array}{cc}
-1 & 0 \\
1 & -0.5
\end{array}\right], N=\left[\begin{array}{cc}
1 & 1 \\
0 & -1
\end{array}\right]
$$

and we can solve

$$
T=\left[\begin{array}{cc}
1 & 0 \\
-1.65 & -0.71
\end{array}\right]
$$

from the Sylvester Equation. However, the nonlinear incremental condition is not satisfied. In fact, the inequality (2.11) 
doesn't always hold, e.g., for $z=\left[\begin{array}{ll}-15 & -2\end{array}\right]^{\top}$ and $d=0$. Moreover, we note the nonlinear incremental condition is used to guarantee that the $z$-subsystem in (2.13) has a certain stability property. In particular, the equilibrium point $z=0$ of the system

$$
\dot{z}=M z-N \psi(z+d)+N \psi(d)
$$

should be GAS. However, the simulation with $z(0)=$

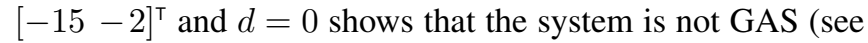
Fig. 1). Therefore, the internal model candidate based on the linear observability condition of the steady state generator doesn't work for this example.

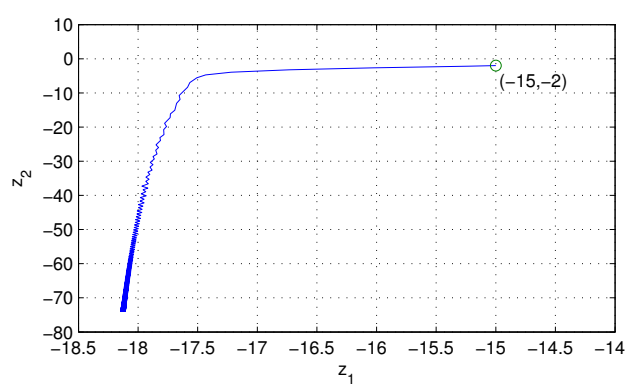

Fig. 1. The trajectory starting from $z=(-15,-2)$ doesn't converge to the equilibrium point $z=0$.

But, we can show that the steady state generator (2.9) is globally detectable. In particular, with the parameters used above, we can solve a diffeomorphism

$$
H(\tau)=\left[\begin{array}{c}
2 \tau_{1}-\sin \tau_{1} \\
-1.65 \tau_{1}-0.71 \tau_{2}
\end{array}\right]
$$

from the differential equation (3.1). We note that the Jacobian matrix of $H(\tau)$ is $T$ given above. With this global delectability property, we don't need to verify the nonlinear incremental condition. To construct the controller, it suffices to verify the stabilization Assumption 2. With a feedforward controller $u=\kappa(x)+\mathbf{u}(v)$, the system (4.1) becomes

$$
\dot{x}=\left[\begin{array}{c}
v_{2} \cos \left(x_{2}+v_{1}\right)-v_{2} \cos v_{1} \\
\sin \left(x_{2}+v_{1}\right)-\sin v_{1}-x_{1}
\end{array}\right]+\kappa(x) .
$$

When $v \in \mathbb{V}$ with $\mathbb{V}$ is compact set, it is easy to find a function $\kappa(x)$ to match the Assumption 2. From Theorem 3.1, a controller exists for the GRORP of the system (4.1). The simulation results are plotted in Fig. 2 including the profiles of the plant state $\xi$, tracking error $e$, and internal model state $\eta$.

\section{CONClusion}

In this paper, we have introduced the concepts of global observability and detectability of the steady state generator in the robust output regulation problem. The concepts are defined in term of a differential equation whose linearized version is the Sylvester Equation. The internal model based on a globally observable/detectable steady state generator is effective for a more general class of nonlinear systems containing non-polynomial uncertainties. Obviously, the
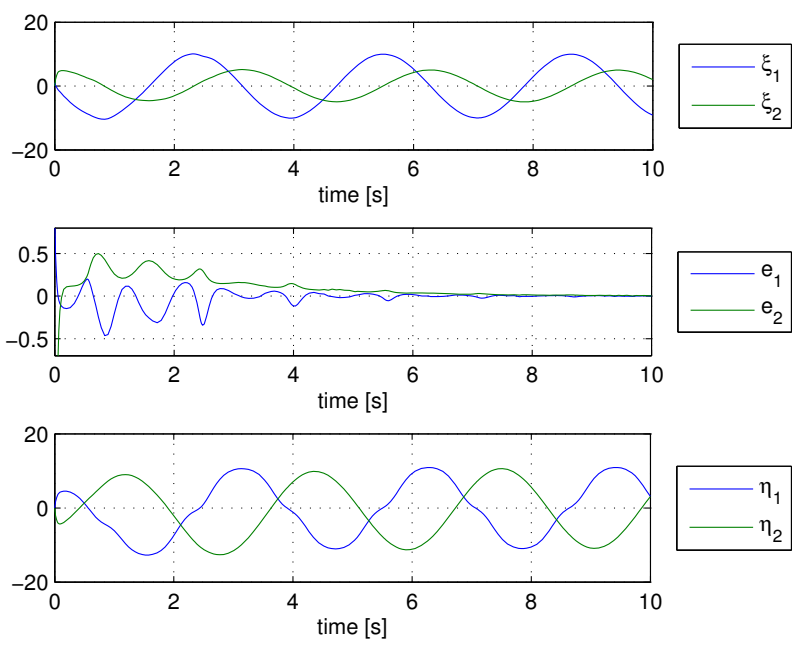

Fig. 2. Profiles of the plant state $\xi$, tracking error $e$, and internal model state $\eta$.

solvability of the differential equation is more complicated than the Sylvester Equation, which is an interesting topic in the future work.

\section{REFERENCES}

[1] C. I. Byrnes, F. Delli Priscoli, A. Isidori, and W. Kang, "Structurally stable output regulation of nonlinear systems," Automatica, vol. 33, pp. 369-385, 1997.

[2] E.J. Davison, "The robust control of a servomechanism problem for linear time-invariant multivariable systems," IEEE Transactions on Automatic Control, vol. 21, pp. 25 - 34, 1976.

[3] B. A. Francis, "The linear multivariable regulator problem," SIAM Journal on Control and Optimization, vol. 15, pp. 486 - 505, 1977.

[4] B.A. Francis and W.M. Wonham, "The internal model principle of control theory," Automatica, vol. 12, pp. 457-465, 1976.

[5] J. Huang, "Remarks on robust output regulation problem for nonlinear systems," IEEE Transactions on Automatic Control, vo1. 46, pp. 20282031, 2001.

[6] J. Huang, Nonlinear output regulation problem: Theory and Applications, Advance in Design and Control, SIAM, 2004.

[7] J. Huang and Z. Chen, "A general framework for tackling the output regulation problem," IEEE Transactions on Automatic Control, No. 12, pp. 2203 - 2218, Dec. 2004.

[8] J. Huang and C-F. Lin, "Internal model principle and robust control of nonlinear systems," Proceedings of the 32nd IEEE Conference on Decision and Control, pp. 1501-1513, December 1993.

[9] A. Isidori and C.I. Byrnes, "Output regulation of nonlinear systems," IEEE Transactions on Automatic Control, vol. 35, pp. 131-140, 1990.

[10] Z. P. Jiang and L. Praly, "Design of robust adaptive controllers for nonlinear systems with dynamic uncertainties," Automatica, vol.34, pp.825-840, 1998.

[11] H. Khalil, "Robust servomechanism output feedback controllers for feedback linearizable systems," Automatica, vol. 30, pp. 1587-1589, 1994.

[12] V.O. Nikiforov, "Adaptive non-linear tracking with complete compensation of unknown disturbances," European Journal of Control, vol. 4, pp. 132-139, 1998.

[13] A. Serrani, A. Isidori, and L. Marconi, "Semiglobal nonlinear output regulation with adaptive internal model," IEEE Transactions on Automatic Control, vol. 46, pp. 1178-1194, 2001.

[14] E. Sontag and A. Teel, "Changing supply function in input/state stable systems", IEEE Transactions on Automatic Control, V40, pp.14761478, 1995. 